\title{
Evaluation of Optic Disc Perfusion with Optical Coherence Tomography Angiography in Acute Non-arteritic Anterior Ischemic Optic Neuropathy
}

\author{
(1) Hatice Kübra Sönmez, () Hatice Arda, (ㄱ) Duygu Gülmez Sevim \\ Erciyes University Faculty of Medicine, Department of Ophthalmology, Kayseri, Turkey
}

\begin{abstract}
Objectives: This study aimed to evaluate superficial peripapillary vascularization qualitatively and quantitatively in patients with acute non-arteritic anterior ischemic optic neuropathy (NAION) using optical coherence tomography angiography (OCT-A).

Materials and Methods: Eleven patients with acute NAION and 14 controls were evaluated retrospectively. Complete ophthalmologic examination with best corrected visual acuity, peripheral visual field test, and disc angiography with OCT-A were performed. Quantitative optic disc perfusion indexes were evaluated by the device with automatically segmentation and qualitative comparison of choroidal, retinal, and en-face peripapillary perfusion angiogram images.

Results: In the NAION and control groups, mean age was $57.55 \pm 12.34$ years and $50.79 \pm 4.67$ years $(\mathrm{p}=0.110)$, the proportion of women was $7 / 11(63.6 \%)$ and $9 / 14(60 \%)$, and best corrected visual acuity was $0.95 \pm 0.63$ and $0.00 \pm 0.0 \operatorname{LogMAR}(\mathrm{p}=0.001$ ), respectively. Visual field defect was present in 10/11 (91\%) eyes in the NAION group. In 6 patients, visual field defects were correlated with areas of peripapillary and optic nerve head hypoperfusion. In the patient group, optic nerve head capillary density was significantly decreased $(\mathrm{p}=0.008)$ and radial peripapillary capillary density was significantly decreased in all sectors except the inferonasal sector.

Conclusion: In our study, we observed that visual field evaluations were partially correlated with optic nerve head and peripapillary capillary perfusion assessed by OCT-A. Being practical and non-invasive, OCT-A is a useful and up-to-date method for evaluating perfusion in NAION.
\end{abstract}

Keywords: Non-arteritic anterior ischemic optic neuropathy, optical coherence tomography angiography, OCT-A

Address for Correspondence: Hatice Kübra Sönmez, Erciyes University Faculty of Medicine, Department of Ophthalmology, Kayseri, Turkey Phone: +90 5439626396 E-mail: drkubrasavasci@gmail.com ORCID-ID: orcid.org/0000-0001-5371-1373

Received: 20.10 .2020 Accepted: 14.04 .2021

Cite this article as: Sönmez HK, Arda H, Gülmez Sevim D. Evaluation of Optic Disc Perfusion with Optical Coherence Tomography Angiography in Acute Nonarteritic Anterior Ischemic Optic Neuropathy. Turk J Ophthalmol 2022;52:30-36

${ }^{\circledR}$ Copyright 2022 by Turkish Ophthalmological Association Turkish Journal of Ophthalmology, published by Galenos Publishing House. 


\section{Introduction}

Anterior ischemic optic neuropathy (AION) is believed to occur most commonly as a result of acute ischemia in the anterior part of the optic nerve. There are two types, nonarteritic and arteritic. ${ }^{1}$ Non-arteritic anterior ischemic optic neuropathy (NAION) is the leading cause of sudden vision loss in the middle-age group. It is painless and generally unilateral, although involvement of the fellow eye may also occur over time. The prevalence of NAION varies by race, particularly in relation to small optic disc size, with a reported incidence of $10.2 / 100,000$ in the United States. ${ }^{2,3}$

The pathogenesis of NAION is known to be associated with impaired blood flow in the posterior ciliary artery (PCA) of the optic nerve head, which results in sudden ischemia in the optic nerve head. ${ }^{4}$

The clinical picture of NAION is often characterized by sudden-onset, painless unilateral vision loss and disc edema with altitudinal visual field defect. This optic neuropathy occurs due to local or systemic predisposing causes. ${ }^{5}$ Among the systemic predisposing causes, conditions such as systemic hypertension and diabetes that cause vasculopathy and end organ damage especially increase the risk of developing NAION. ${ }^{6,7,8}$ Similarly, causes of decreased optic disc perfusion such as nocturnal hypotension and obstructive sleep apnea syndrome have been associated with NAION. ${ }^{9,10}$

Choroidal perfusion assessed by fluorescein angiography is classically preserved in NAION. ${ }^{11}$ However, some studies have demonstrated thinning of the subfoveal or peripapillary choroidal layer in the acute and chronic phases, indicating that a thinner choroid may also be a risk factor. ${ }^{12,13}$

Optical coherence tomography angiography (OCT-A) is a new, non-invasive imaging method that can be used to visualize the microcirculation in the retina and around the optic nerve. ${ }^{5}$ Unlike fundus fluorescein angiography, it requires no exogenous contrast agent. OCT-A enables quantitative evaluation of peripapillary retinal, choroidal, and optic nerve head capillary density and flow. ${ }^{14}$

This study aimed to qualitatively and quantitatively evaluate superficial peripapillary vascularization using OCT-A and thereby non-invasively visualize ischemia in patients with acute NAION.

\section{Materials and Methods}

\section{Patient Selection and Study Design}

This study included 11 patients with a diagnosis of NAION who presented to the University of Erciyes Ophthalmology Clinic between January and July 2019, and 14 healthy volunteers (control group) with completely normal ophthalmological examination findings. The 11 patients presented with sudden- onset painless vision loss for less than 2 weeks on one side $(n=11$ eyes). Clinical examinations were performed by two different ophthalmologists and the diagnosis was made by excluding other causes of optic neuropathy. All patients underwent neurological evaluation and neurological imaging to exclude central causes. None of the eyes included in the study had a previous diagnosis of NAION. All patients who met the NAION diagnostic criteria and cooperated with the tests were included in the study. Exclusion criteria were: refractive error greater than \pm 4 diopters, optic disc anomalies, retinopathy, intraocular pressure higher than $21 \mathrm{mmHg}$ and glaucoma diagnosis, use of medications that affect the optic nerve (digoxin, vigabatrin, ethambutol), history of eye trauma and/or retinal surgery, and history of cataract surgery within the last 6 months.

\section{Clinical Examinations}

After obtaining past and present clinical history, best corrected visual acuity (BCVA) (assessed with Snellen chart and converted to $\log$ MAR equivalent), intraocular pressure measurement, slit-lamp anterior segment examination, relative afferent pupil defect (RAPD) examination with swinging flashlight test, color vision examination with Ishihara test, and fundoscopic examination with $90 \mathrm{D}$ lens were performed. Refraction was measured using an autorefractometer (TonoRef II, Nidek, Japan). C-reactive protein level, erythrocyte sedimentation rate, biochemical markers, and complete blood count were performed to exclude arteritic AION. Orbital and cranial magnetic resonance imaging was performed in all patients to rule out central and retrobulbar etiology. Pattern visual evoked potential (VEP) test was performed (Metrovision Vision Monitor Mon2018F, 2018). Each patient and control subject underwent $30^{\circ}$ peripheral visual field test (Octopus-900, Haag-STEIT eyesuite static perimetry, V2.2.0) and disc angiography using OCT-A (RTVue XR Avanti Optovue Inc, Fremont, CA, version 2017.1.0.151).

\section{OCT-A Imaging}

All OCT-A measurements were made by the same ophthalmologist at the same time of day (10:00-12:00) to minimize diurnal changes. In OCT-A using the split-spectrum amplitude correlation angiography algorithm, 70,000 A-sections per second were acquired and measurements were evaluated from $4.5 \times 4.5 \mathrm{~mm}$ disc images with a tissue axial resolution of 5 $\mu \mathrm{m} .{ }^{15}$ Measurements obtained from OCT-A images with signal strength below 6 , motion artifacts due to poor fixation, and segmentation errors were excluded from the study.

Quantitative optic disc perfusion values were measured automatically by the device software. The device automatically determined peripapillary vascular density (\%) in 10 segments and retinal nerve fiber layer (RNFL) measurements $(\mu \mathrm{m})$. Qualitative peripapillary perfusion was evaluated by comparing choroidal, retinal, and en-face angiogram images. Optic disc 
head and peripapillary measurements were determined for the vitreous/retinal layer over the outer plexiform layer, the radial peripapillary capillary (RPC) layer between the inner limiting membrane and the RNFL, and the choroidal layer under the retinal pigment epithelium (RPE), which were automatically segmented by the software. ${ }^{16}$

Statistical Analysis

SPSS version 22.0 (IBM Corp, Armonk, NY, USA) was used for analyses. Data distribution characteristics were evaluated using the Shapiro-Wilk test. Normally distributed quantitative data were expressed as mean \pm standard deviation and categorical data as percentage (\%). Independent-samples t-test was used to compare quantitative data that showed normal distribution. Categorical data were compared using chi-square (Fisher's Exact) test. A p-value $<0.05$ was considered statistically significant.

\section{Ethical Considerations}

The study was approved by the Erciyes University Faculty of Medicine Clinical Trials Ethics Committee (approval number: 2019/139).

\section{Results}

The study included 11 eyes of 11 NAION patients (group 1 ) and 14 randomly selected eyes of 14 healthy volunteers (group 2). The mean ages of the groups were $57.55 \pm 12.34$ and $50.79 \pm 4.67$ years, respectively $(\mathrm{p}=0.110)$. The proportion of women was $63.6 \%(7 / 11)$ in group 1 and 60\% (9/14) in group 2. NAION episodes were primary in all affected eyes. Two patients in group 1 had concomitant diabetes and hypertension. No signs of retinopathy were observed in these patients. The demographic data and rates of hypertension/diabetes of the groups are summarized in Table 1.

BCVA was 0.95 $\pm 0.63 \log$ MAR equivalent in affected eyes in group 1 , compared to $0.00_{ \pm} 0.0$ in group $2(\mathrm{p}=0.001)$. In group 1 , RAPD was present in $4(36.4 \%)$ of 11 eyes but could not be observed in the 7 eyes with signs of previous optic neuropathy in the fellow eye. Color vision was impaired in 10 (91\%) of the 11 eyes. Attenuated wave amplitudes and prolonged P100 latencies were observed on VEP 60'.
Visual field defect was present in $10(91 \%)$ of the eyes (peripheral concentric narrowing in 2 eyes, inferior-hemi scotoma in 4, superior-temporal scotoma in 1, inferior-temporal scotoma in 1, paracentral scotoma in 1 , and inferior-nasal scotoma in 1 eye); only 1 eye did not have a localized scotoma but showed reduced sensitivity in the central and temporal regions.

RPC hypoperfusion was defined as dark areas where the capillary network was not visible on OCT-A images. Hypoperfusion in the RPC was not observed in 1 eye and was observed in the optic disc head in 1 eye, in at least 2 quadrants in 4 eyes, in 4 quadrants in 2 eyes, and in 1 quadrant in 3

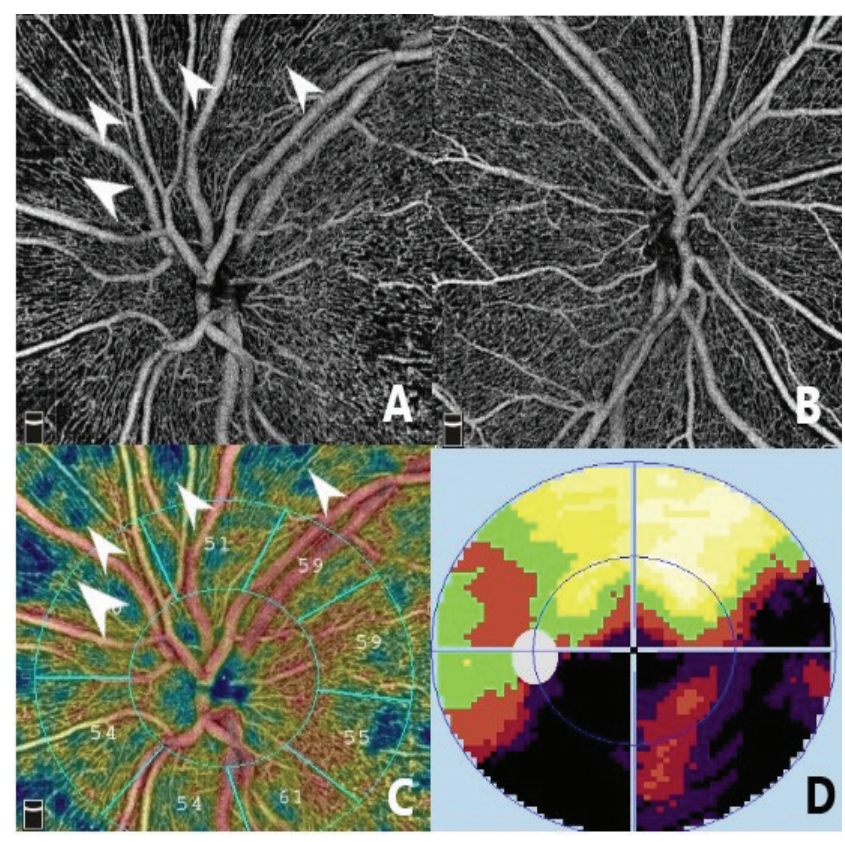

Figure 1. A 49-year-old woman presented with loss of vision in the left eye for 5 days. A) In the optical coherence tomography angiography (OCT-A) radial peripapillary capillary (RPC) cross-section, hypoperfusion is observed in en face images as dark areas in the superior nasal region, indicated by white arrowheads. B) No perfusion defect is observed in the cross-section obtained in the superficial OCT-A section, corresponding to above the outer plexiform layer. C) Color mapping of the vascular density in the RPC layer section indicates reduced perfusion in the blue areas indicated by the white arrowheads. This supports the presence of hypoperfusion consistent with the en face images. D) Visual field shows a scotoma in the inferior-hemi section corresponding to the predominantly inferior hypoperfused region

Table 1. Comparison of selected demographic data and visual field values

\begin{tabular}{|l|l|l|l|}
\hline & $\begin{array}{l}\text { NAION, } \\
\text { group 1 (n=11) }\end{array}$ & $\begin{array}{l}\text { Control, } \\
\text { group 2 (n=14) }\end{array}$ & p \\
\hline Age (years), mean \pm SD & $57.56 \pm 12.34$ & $50.79 \pm 4.67$ & 0.110 \\
\hline Sex (female), n (\%) & $7(63.6)$ & $9(60.0)$ & 0.604 \\
\hline DM, $n(\%)$ & $2(18.2)$ & 0 & - \\
\hline Hypertension, $n(\%)$ & $3(27.3)$ & 0 & - \\
\hline Visual field defect, n (\%) & $8(72.3)$ & 0 & - \\
\hline NAION: Non-arteritic acute ischemic optic neuropathy, SD: Standard deviation, DM: Diabetes mellitus & \\
\hline
\end{tabular}


eyes, predominantly in the temporal quadrant. Figure 1 shows a visual field defect corresponding to an area of hypoperfusion detected on OCT-A in a patient with inferior hemiscotoma (Figures 1a-d). Figure 2 shows an inferior-temporal visual field defect corresponding to a hypoperfused area observed on OCT-A imaging in another patient (Figures 2a-d).

In 6 patients, visual field defects corresponded to regions with peripapillary and optic disc head hypoperfusion. One patient exhibited no hypoperfusion. In the other 4 patients, the hypoperfused areas and visual field defects were not correlated.

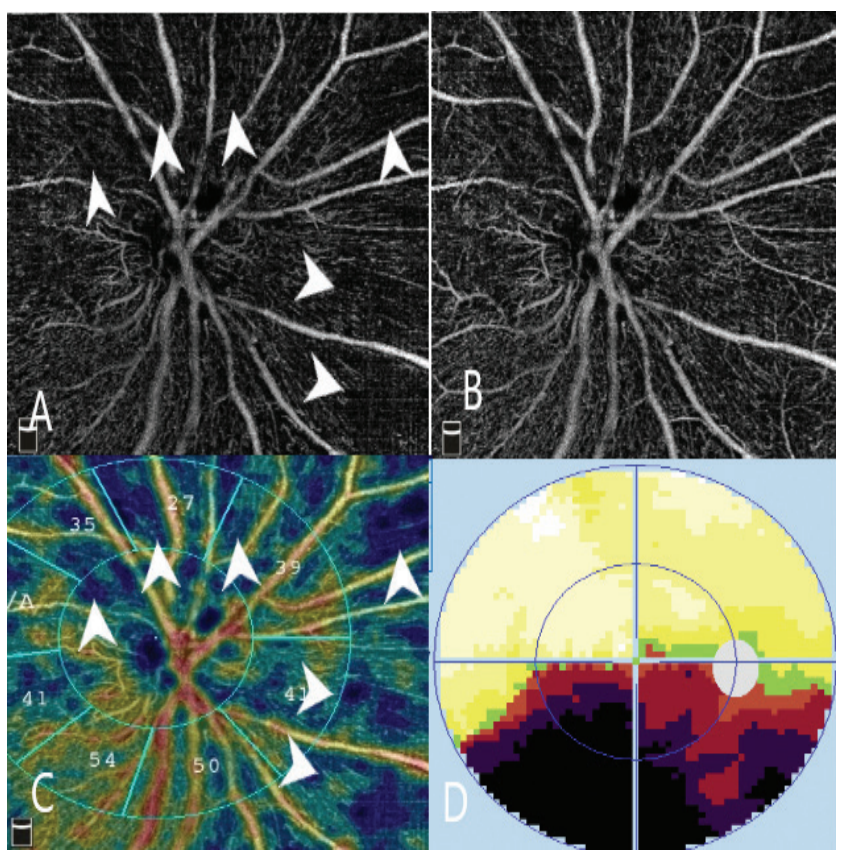

Figure 2. A 60-year-old woman had vision loss in her right eye for 1 week. A) On OCT-A imaging, dark areas consistent with hypoperfusion (white arrowheads) are observed in the RPC layer en face measurement cross-section. B) No disruption of vascular flow is observed in the superficial OCT-A cross-section above the outer plexiform layer. C) The vascular density color map of the RPC layer shows predominantly superior nasal hypoperfused and non-perfused areas corresponding to the dark areas on en face images (white arrowheads). D) Visual field demonstrates scotoma areas in the form of an altitudinal defect in the inferior hemisphere extending temporally, representing the hypoperfused areas

OCT-A: Optical coherence tomography angiography, RPC: Radial peripapillary capillary
In group 1, the RPC appeared mildly dilated and tortuous on OCT-A compared to the control group and unaffected fellow eyes.

In the quantitative analysis of OCT-A measurements, there was a statistically significant difference in RPC densities when the two groups were compared $(\mathrm{p}=0.005)$. There was also a significant difference in peripapillary RNFL measurements, with larger values in the patient group $(\mathrm{p}=0.008)$. RPC and optic disc head capillary densities were lower in the patient group compared to the control group. When combined, the difference between the groups was significant $(\mathrm{p}=0.008)$ while optic disc vascular density measurements were not statistically significantly different ( $\mathrm{p}=0.052)$. The patients' RNFL, RPC, and optic disc head capillary densities are summarized in Table 2.

In the comparison of RPC density in the 10 different sectors, the patient group had significantly lower values in all sectors except the inferior sector $(\mathrm{p}=0.115)$ (Table 3).

\section{Discussion}

NAION occurs as a result of sudden ischemia due to impaired PCA circulation in the optic disc head. Two causes are emphasized in its etiology and pathogenesis. The first and most common is non-thromboembolic transient non-perfusion or hypoperfusion of the PCA. This hypotension, which may occur as systemic fluctuation, or sudden intraocular pressure elevations can lead to transient malperfusion of the PCA. The second, less common cause is embolic events in the arteries and arterioles that supply the optic nerve head. NAION resulting from multiple embolisms can cause more severe and permanent vision loss. ${ }^{4}$ Kavuncu et al. ${ }^{17}$ reported that visual prognosis was worse in older age and those with systemic predisposing factors, while patients with involvement at a younger age had better visual outcomes. In another study, it was found that NAION patients had electrophysiological changes suggestive of axonal loss in the unaffected eye, which the authors defended with the notion that axonal loss caused by ischemia may extend to the optic chiasm and affect the fellow eye. ${ }^{18}$

A study evaluating acute arteritic and non-arteritic AION patients using OCT-A showed that optic disc head and RPC

Table 2. Comparison of peripapillary thickness and optic disc vascular density measurements between the groups

\begin{tabular}{|l|l|l|l|}
\hline & $\begin{array}{l}\text { NAION, } \\
\text { group 1 (n=11) } \\
\text { Mean } \pm \text { SD }\end{array}$ & $\begin{array}{l}\text { Control, } \\
\text { group 2 (n=14) } \\
\text { Mean } \pm \text { SD }\end{array}$ & p \\
\hline RNFL $(\mu \mathrm{m})$ & $220.64 \pm 104.91$ & $116.64 \pm 11.60$ & 0.008 \\
\hline Peripapillary capillary density $(\%)$ & $44.65 \pm 8.54$ & $53.95 \pm 1.68$ & 0.005 \\
\hline Optic disc head density (\%) & $43.90 \pm 5.84$ & $48.49 \pm 5.32$ & 0.052 \\
\hline All capillary density (\%) & $43.72 \pm 6.18$ & $49.97 \pm 2.59$ & 0.008 \\
\hline NAION: Non-arteritic acute ischemic optic neuropathy, SD: Standard deviation, RNFL: Retinal nerve fiber layer. Statistically significant values are highlighted in bold \\
\hline
\end{tabular}


Table 3. Comparison of segmental radial peripapillary capillary density measurements (\%) between the groups

\begin{tabular}{|l|l|l|l|}
\hline Segments & $\begin{array}{l}\text { NAION, } \\
\text { group 1 (n=11) } \\
\text { Mean } \pm \text { SD }\end{array}$ & $\begin{array}{l}\text { Control, } \\
\text { group 2 (n=14) } \\
\text { Mean } \pm \text { SD }\end{array}$ & p \\
\hline Superior-hemi & $41.7 \pm 10.54$ & $53.57 \pm 1.89$ & 0.004 \\
\hline Inferior-hemi & $45.59 \pm 9.51$ & $54.30 \pm 2.19$ & 0.013 \\
\hline Nasal superior & $41.17 \pm 10.10$ & $50.57 \pm 3.33$ & 0.012 \\
\hline Nasal inferior & $42.39 \pm 9.84$ & $51.38 \pm 3.44$ & 0.014 \\
\hline Inferior nasal & $46.16 \pm 12.09$ & $52.59 \pm 3.41$ & 0.115 \\
\hline Inferior temporal & $50.60 \pm 10.90$ & $61.09 \pm 2.79$ & 0.010 \\
\hline Temporal inferior & $45.27 \pm 9.24$ & $54.77 \pm 2.79$ & 0.007 \\
\hline Temporal superior & $47.55 \pm 9.99$ & $57.87 \pm 1.60$ & 0.007 \\
\hline Superior temporal & $44.98 \pm 11.45$ & $57.75 \pm 1.85$ & 0.004 \\
\hline Superior nasal & $40.17 \pm 12.30$ & $50.8 \pm 3.94$ & 0.018 \\
\hline NAION: Non-arteritic acute ischemic optic neuropathy, SD: Standard deviation. Statistically significant values are highlighted in bold & \\
\hline
\end{tabular}

density was decreased in acute NAION patients compared to healthy controls. In addition, non-perfusion areas in the optic disc head detected by indocyanine green angiography and fundus fluorescein angiography were correlated with the non-perfusion areas seen on OCT-A, and it was concluded that OCT-A evaluation provided useful information in the diagnosis of NAION. ${ }^{19}$ In another study, qualitative OCT-A evaluation of the optic disc head in NAION revealed increased capillary tortuosity and dilation, predominantly in the hypoperfused areas, in more than half of the patients. The authors suggested this could be explained by the hypothesis of venous insufficiency in the optic disc head secondary to temporary hypoperfusion or venous infarction. ${ }^{20}$ In the literature, it has been reported that eyes with NAION have greater involvement in the temporal quadrant due to choroidal watershed zone damage. ${ }^{14,21}$ Sharma et al. ${ }^{5}$ observed increased capillary tortuosity in patients with acute NAION and attributed it to transient venous insufficiency. In our study, quantitative RPC segmental measurements were significantly reduced in all sectors except the inferonasal sector. In qualitative peripapillary OCT-A evaluations, hypoperfusion was not observed in 1 patient but was detected in 1 or more quadrants (including the temporal quadrant) in 8 patients, consistent with the literature. However, the decrease in capillary density measured by OCT-A may also be a result of edematous compression of the optic disc or secondary signal attenuation due to the shadowing effect of the fluid (edema, hemorrhage). One study demonstrated that in patients with decreased peripapillary flow densities, low flow persisted in repeated OCT-A measurements after resolution of optic disc edema. ${ }^{5}$ In our OCT-A evaluation, we found that RNFL values were higher in the patient group than the control group, contrary to the literature. A previous study showed that in animal models of ischemic optic neuropathy, more severe optic nerve ischemia in the acute phase leads to more optic disc edema and vision loss. ${ }^{22}$ Measured RNFL values may have been higher due to the fact that our patient group had optic disc edema associated with acute NAION.

In our study, we determined that visual field assessments were partially correlated with optic disc head and RPC perfusion evaluated by OCT-A, with one patient having no hypoperfusion area detected on OCT-A and no detectable visual field defect. Six patients had predominantly inferior visual field defects that were consistent with the hypoperfused optic disc head and peripapillary quadrants. However, four patients' visual field defects did not correspond to the areas of hypoperfusion observed in RPC images on OCT-A scans. Rougier et al. ${ }^{23}$ reported poor correlation between OCT-A measurements and visual field in their study, observing close correlation between the hypoperfused area and visual field defect pattern in only one patient. They argued that visual field defects were associated with changes in PCA flow rather than peripapillary flow. Ling et al. ${ }^{24}$ evaluated optic disc perfusion and determined that visual acuity and visual field defects were correlated with hypoperfusion detected on OCT-A. In another study, visual field defects were reported to be significantly correlated with optic disc vascular density, vascular tortuosity, and RNFL thicknesses. ${ }^{25}$ Gaier et al. ${ }^{26}$ concluded that the pattern of visual field defect in patients with acute and non-acute NAION correlated with associated regions of reduced patent capillary density. Consistent with the literature, we found that BCVA was lower in the patient group in our study.

OCT-A is a current, practical, and non-invasive method that can be easily applied to support the diagnosis of NAION and is 
useful in the qualitative and quantitative assessment of retinal and choroidal vascularization.

\section{Study limitations}

This study has certain limitations. One of these is the small number of patients included in the study. In addition, we could not perform manual segmentation due to the absence of the most recent version of Angiovue in the Optovue software. Therefore, calculations were based on the inner and outer segments determined automatically by the device. Other limitations were the small measurement area and limited evaluation of the peripapillary area, and the fact that scans were not repeated after edema resolution to rule out possible RPC circulatory impairment caused by obstruction, which can also cause optic disc edema.

\section{Conclusion}

The results of this study indicate that optic disc head and superficial peripapillary microcirculation are impaired in acute NAION. The partial correlation between detected microvascular changes and visual field defects reveals an important structural and functional relationship.

In summary, OCT-A is a current, rapid, and non-invasive method for the assessment of the peripapillary microcirculation in NAION patients. Controlled randomized studies with larger patient numbers are necessary to more clearly demonstrate the relationship between visual field defects and the microcirculatory impairments detected by OCT-A.

Ethics

Ethics Committee Approval: The study was approved by the Erciyes University Faculty of Medicine Clinical Trials Ethics Committee (approval number: 2019/139). Informed Consent: Retrospective study.

Peer-review: Externally peer-reviewed.

\section{Authorship Contributions}

Surgical and Medical Practices: H.K.S., D.G.S., H.A., Concept: H.K.S., D.G.S., H.A., Design: H.K.S., D.G.S., H.A., Data Collection or Processing: H.K.S., D.G.S., Analysis or Interpretation: H.K.S., D.G.S., H.A., Literature Search: H.K.S., D.G.S., H.A., Writing: H.K.S., D.G.S., H.A.

Conflict of Interest: No conflict of interest was declared by the authors.

Financial Disclosure: The authors declared that this study received no financial support.

\section{References}

1. Characteristics of Patients With Nonarteritic Anterior Ischemic Optic Neuropathy Eligible for the Ischemic Optic Neuropathy Decompression Trial. Arch Ophthalmol. 1996;114:1366-1374.
2. Jonas JB. Optic disc morphology and NAAION. Br J Ophthalmol. 2009;93:703.

3. Johnson LN, Arnold AC. Incidence of nonarteritic and arteritic anterior ischemic optic neuropathy. Population-based study in the state of Missouriand Los Angeles County, California. J Neuroophthalmol. 1994;14:38-44.

4. Hayreh SS. Ischemic optic neuropathy. Prog Retin Eye Res. 2009;28:34-62.

5. Sharma S, Ang M, Najjar RP, Sng C, Cheung CY, Rukmini AV, Schmetterer L, Milea D. Optical coherence tomography angiography in acutenon-arteritic anterior ischaemic optic neuropathy. Br J Ophthalmol. 2017;101:1045-1051.

6. Cestari DM, Gaier ED, Bouzika P, Blachley TS, De Lott LB, Rizzo JF, Wiggs JL, Kang JH, Pasquale LR, Stein JD. Demographic, Systemic, and Ocular Factors Associated with Nonarteritic Anterior Ischemic Optic Neuropathy. Ophthalmology. 2016;123:2446-2455.

7. Lee MS, Grossman D, Arnold AC, Sloan FA. Incidence of nonarteritic anterior ischemic optic neuropathy: increased risk among diabetic patients. Ophthalmology. 2011;118:959-963.

8. Hayreh SS, Joos KM, Podhajsky PA, Long CR. Systemic diseases associated with nonarteritic anterior ischemic optic neuropathy. Am J Ophthalmol. 1994;118:766-780.

9. Hayreh SS, Zimmerman MB, Podhajsky P, Alward WL. Nonarteritic anterior ischemic optic neuropathy: role of nocturnal arterial hypotension. Arch Ophthalmol. 1997;115:942-945.

10. Wu Y, Zhou LM, Lou H, Cheng JW, Wei RL. The Association Between Obstructive Sleep Apnea and Nonarteritic Anterior Ischemic Optic Neuropathy: A Systematic Review and Meta-Analysis. Curr Eye Res. 2016; 41:987-992.

11. Arnold AC, Hepler RS. Fluorescein angiography in acute nonarteritic anterior ischemic optic neuropathy. Am J Ophthalmol. 1994;117:222-230.

12. Schuster AK, Steinmetz P, Forster TM, Schlichtenbrede FC, Harder BC, Jonas JB. Choroidal thickness in nonarteritic anterior ischemic optic neuropathy. Am J Ophthalmol. 2014;158:1342-1347.e1.

13. García-Basterra I, Lahrach I, Morillo Sánchez MJ, Kamal-Salah R, RíusDíaz F, Dawid Milner MS, García-Campos JM. Analysis of peripapillary choroidal thickness in non-arteritic anterior ischaemic optic neuropathy. $\mathrm{Br} \mathrm{J}$ Ophthalmol. 2015;100:891-896.

14. Rebolleda G, Díez-Álvarez L, GarcíaMarín Y, de Juan V, Muñoz-Negrete FJ. Reduction of Peripapillary Vessel Density by Optical Coherence Tomography Angiography from the Acute to the Atrophic Stage in Non-Arteritic Anterior Ischaemic Optic Neuropathy. Ophthalmologica. 2018;240:191-199.

15. Chalam KV, Sambhav K. Optical coherence tomography angiography in retinal diseases. J Ophthalmic Vis Res. 2016;11:84-92.

16. Huang D, Jia Y, Gao SS. Interpretation of optical coherence tomography angiography. Practicalhandbook of OCT angiography, 6, 2016.

17. Kavuncu S, Nalçacioğlu P, İlhan B, Budakoğlu Ö. Bir Üçüncü Basamak Göz Hastanesinde İzlenen Non-arteritik Ön İskemik Optik Nöropatili Hastaların Klinik ve Demografik Özellikleri. Turkiye Klinikleri J Ophthalmol. 2020;29:7-18.

18. Biler ED, Kaya E, Afrashi F, Üretmen Ö. Pattern visual evoked potentials in the fellow eye of the patients with unilateral non-arteritic anterior ischemic optic neuropathy. Turk J Ophthalmol. 2014;44:15-18.

19. Balducci N, Morara M, Veronese C, Barboni P, Casadei NL, Savini G, Parisi V, Sadun AA, Ciardella A. Optical coherence tomography angiography in acute arteritic and non-arteritic anterior ischemic optic neuropathy. Graefes Arch Clin Exp Ophthalmol. 2017;255:2255-2261.

20. Levin LA, Danesh-Meyer HV. Hypothesis: a venous etiology for nonarteritic anterior ischemic optic neuropathy. Arch Ophthalmol. 2008;126:1582-1585.

21. Hayreh SS. Posterior ciliary artery circulation in health and disease: the Weisenfeldlecture. Invest Ophthalmol Vis Sci. 2004;45:749-748.

22. Johnson MA, Miller NR, Nolan T, Bernstein SL. Peripapillary Retinal Nerve Fiber Layer Swelling Predicts Peripapillary Atrophy in a Primate Model of Nonarteritic Anterior Ischemic Optic Neuropathy. Invest Ophthalmol Vis Sci. 2016;57:527-532. 
23. Rougier MB, Delyfer MN, Korobelnik JF. [OCT angiography of acutenonarteritic anterior ischemic optic neuropathy]. J Fr Ophtalmol. 2017;40:102109.

24. Ling JW, Yin X, Lu QY, Chen YY, Lu PR. Optical coherence tomography angiography of optic disc perfusion in non-arteritic anterior ischemic optic neuropathy. Int J Ophthalmol. 2017;10:1402-1406.

25. Pierro L, Arrigo A, Aragona E, Cavalleri M, Bandello F. Vessel Density and Vessel Tortuosity Quantitative Analysis of Arteritic and Non-arteritic
Anterior Ischemic Optic Neuropathies: An Optical Coherence Tomography Angiography Study. J Clin Med. 2020;9:1094.

26. Gaier ED, Wang M, Gilbert AL, Rizzo JF, Cestari DM, Miller JB. Quantitative analysis of optical coherence tomographic angiography (OCT-A) in patients with non-arteritic anterior ischemic optic neuropathy (NAAION) corresponds to visual function. PLoS One. 2018;13:e0199793. 